Am J Clin Oncol. 2018 November ; 41(11): 1125-1131. doi:10.1097/COC.0000000000000433.

\title{
Targeting tumor metabolism with statins during treatment for advanced-stage pancreatic cancer
}

\author{
Nick A. larrobino, B.Sc. ${ }^{1}$, Beant Gill, M.D. ${ }^{2}$, Mark E. Bernard, M.D. ${ }^{3}$, Mark V. Mishra, M.D. ${ }^{4}$, \\ Colin E. Champ, M.D. ${ }^{2}$ \\ ${ }^{1}$ University of Pittsburgh School of Medicine, Pittsburgh, PA \\ ${ }^{2}$ Department of Radiation Oncology, University of Pittsburgh Medical Center, Pittsburgh, PA \\ ${ }^{3}$ Department of Radiation Medicine, University of Kentucky, Lexington, KY \\ ${ }^{4}$ Department of Radiation Oncology, University of Maryland School of Medicine, Baltimore, MD
}

\section{Abstract}

Introduction-A growing body of preclinical data suggests that statins may exert potent antitumor effects, yet the interactions of these medications with standard therapies and clinical outcomes in this population is less clear. We assessed the impact of statin use on outcomes in patients with advanced-stage pancreatic adenocarcinoma undergoing various treatments.

Method and Materials-After IRB approval, we conducted a retrospective-cohort study consisting of 303 newly diagnosed advanced-stage pancreatic adenocarcinoma patients to determine the impact of statin use on outcomes. Univariate and multivariable Cox proportional hazard regression models were utilized to estimate hazard ratios. Time-to-event was estimated using Kaplan-Meier survival analysis for overall survival, distant metastasis, and locoregional failure. Baseline and active statin usage were assessed and to mitigate risk of immortal time bias, sub-analysis excluding patients with under 6 months of follow-up was conducted.

Results-Both prior $(p=0.021)$ and active $(p=0.030)$ statin usage correlated with improved survival in this cohort. Surgery, chemoradiation, and statin use improved 2-year survival rates ( 84.1 vs. $55.0 \%, p<0.001)$. On multivariable analysis, statin exposure was associated with overall survival (HR 0.662, $p=0.027$ ) and trended to significance for freedom from distant metastasis (HR $0.577, p=0.060$ ). Comorbid conditions were not significantly associated with outcomes.

Conclusions-Statin use was associated with improved overall survival in advanced-stage pancreatic adenocarcinoma patients. This data supports previous findings in early-stage pancreatic adenocarcinoma and other cancer sites. To our knowledge this is the first report to examine the efficacy of statin use as a supplementary treatment option in advanced-stage pancreatic adenocarcinoma patients.

Corresponding Author: Colin E. Champ, M.D., University of Pittsburgh Medical Center, Department of Radiation Oncology, 815 Freeport Road, Pittsburgh, PA 15215, Phone: (412) 784-4900, Fax: (412) 784-4905, champce@ upmc.edu.

Conflicts of Interest: None declared 


\section{Keywords}

pancreatic cancer; statins; radiation therapy; chemotherapy; immunotherapy

\section{Introduction}

Pancreatic cancer is the 4 th leading cause of cancer mortality in the United States. ${ }^{1}$

Although mortality rates continue to decline at other major cancer sites such as colorectal, breast, and prostate, minimal improvements have been made with pancreatic cancer. Currently, surgical resection followed by adjuvant treatment represents the only potential curative treatment option. However, only $20-30 \%$ of pancreatic cancer patients are candidates for curative resection due to advanced disease at presentation. ${ }^{2}$ Even among patients undergoing surgical resection, they remain at high risk for local and/or distant recurrence, ${ }^{3}$ illustrating the need for advancements in treatment options.

Cholesterol is a critical component of the mammalian membrane lipid bilayer and is therefore fundamentally required for cell growth and proliferation. Statins, a widely prescribed group of hyperlipidemia medications, are known to inhibit 3-hydroxy-3methylglutaryl coenzyme A (HMG-CoA reductase), thus reducing cholesterol biosynthesis and serum lipids. Given their efficacy in reducing cholesterol availability, statins have been hypothesized to impede tumorigenesis and disease metastasis. ${ }^{4}$ By reducing downstream products of the mevalonate pathway, statins have been shown to alter protein expression consistent with pro-apoptotic and anti-proliferative states, $, 5,6$ and increase intracellular reactive oxygen species (ROS) production, leading to cytotoxic effects in cancer cells. ${ }^{7}$ Experimental studies have further suggested statin medications may hinder the progression from $\mathrm{G}_{1}-\mathrm{S}$ phase in cell cycle progression, highlighting potential radiosensitizing effects. ${ }^{4}$ Therefore, baseline statin usage before and after primary cancer diagnosis has been hypothesized to improve cancer-specific outcomes.

Although preclinical data is promising, clinical data to support the hypothesized chemopreventive effects against cancer are scant. ${ }^{8,9}$ In lieu of these findings, some have speculated that statins may have effects in preventing disease progression and synergizing with other cancer treatments, rather than impeding tumorigenesis. Indeed, several studies thus far have linked statin use to improved cancer outcomes at numerous sites. ${ }^{10-12}$ However, to our knowledge only two studies have examined the impact statin use may have on survival in pancreatic cancer patients, both noting significantly improved overall survival in early stage disease. ${ }^{13,14}$

In the present study, we examine the relationship between statin use before, during, and after cancer diagnosis, and overall survival, loco-regional failure, and distant metastasis. We hypothesized that baseline statin use would correlate with improved cancer-specific outcomes. 


\section{Material and Methods}

\section{Patient Population}

After institutional review board approval, we retrospectively evaluated the medical records of all patients with newly diagnosed pancreatic adenocarcinoma and treated with radiation therapy at the University of Pittsburgh Hillman Cancer Center between 2004 and 2014. Due to institutional practice preference, all patients received stereotactic body radiation (SBRT) in single or three-fraction regimens as monotherapy or combination treatment with surgery and systemic therapy. In cases of multi-modality therapy, radiation was delivered either adjuvantly or neoadjuvantly with surgery and/or chemotherapy at the discretion of the primary treatment team.

\section{Measures and Exposure Data}

Patient and disease characteristics including age, race, gender, comorbidities, medications, pre-radiation therapy body mass index (BMI), lymph node status, and treatment protocol were collected from electronic medical records. In accordance with previous work ${ }^{13}$ and to better evaluate the impact of duration/timing of exposure, we further classified baseline statin exposure as usage prior to or at diagnosis and active usage as statin dispensation after primary cancer diagnosis. This method represents a practical manner to help elucidate if statins may influence outcomes in this study cohort by systemic effects, local synergistic effects with radiation therapy, or not at all.

Prior studies have quantified daily statin dosages in an attempt to establish a dose-response relationship between statin medications and outcomes. ${ }^{11}$ We collected available simvastatin and atorvastatin dosages, standardized to simvastatin equivalents, and stratified dosages into interval intensity groups. Unfortunately, statin dosages were infrequently recorded and were therefore not included for analysis. Considering most prior statin users were also active users $(91.5 \%)$ in our cohort, an additional benefit of stratifying statin use chronologically is that this approach allows us to analyze the effect cumulative statin dose may have on our primary outcomes of interest. Furthermore, the use of a lag period in which medication users switch from the unexposed to the exposed group after a predetermined duration of medication use is recommended to reduce reverse causation bias. ${ }^{15}$ The electronic medical record, PowerChart (Cerner, UK), records the date of medication prescription and cessation. Given that similar studies have used 6 months as an appropriate lag period, ${ }^{16}$ we excluded patients from the statin group if they discontinued statin medications prior to 6 months of exposure.

\section{Statistical Analysis}

Primary outcomes assessed included overall survival, loco-regional recurrence, and distant metastases. Given the poor prognosis of pancreatic cancer, we found overall survival to be an appropriate measure of cancer mortality. Local progression was defined using RECIST 1.1 criteria, with an increase of at least $20 \%$ in sum of tumor diameters and an absolute increase of $\geq 5 \mathrm{~mm}$ was considered as progressive disease. ${ }^{17}$ Disease progression to regional lymph nodes identified as $\mathrm{n} 1, \mathrm{n} 2$, or $\mathrm{n} 3$ in JPS classification or new pancreatic malignancy outside of the radiation field was characterized as regional failure. Loco-regional recurrence was defined as the presence of local failure, regional failure, or both during the study period. 
We conducted Kaplan-Meier survival analyses to estimate the time-to-event for each of our primary outcomes. Select variables were analyzed in univariate survival analysis, including patient characteristics, medications, comorbidities, treatment protocol, and lymph node status. Variables that attained or approached significance $(p=0.10)$ on univariate survival analysis were considered for multivariable Cox regression. Given the number of events, multivariable Cox proportional-hazards regression analyses was completed including all variables with $p<0.10$ as a generous cut-off in addition to those of clinical interest or significance. Data was analyzed using IBM SPSS Statistics Version 23 (IBM, Armonk, NY).

\section{Results}

\section{Patient Demographics}

We identified 308 patients with advanced-stage pancreatic cancer treated between 2004 and 2014. Patients with non-adenocarcinoma histology $(n=5)$ were excluded, and the remaining 303 patients were included for analysis. Our study cohort was composed of a balanced proportion of males and females, at $152(50.2 \%)$ and 151 (49.8\%), respectively. Approximately half (44.9\%) of patients underwent surgical resection, the majority received chemotherapy (81.8\%), and a small group received immunotherapy (4.0\%). Among our study cohort, 59 (19.5\%) patients were identified as statin users at or before diagnosis and $71(23.4 \%)$ as active statin users after diagnosis. As previously alluded to, among the 59 patients on statin medications at or before the time of diagnosis, $54(91.5 \%)$ continued statin therapy after cancer diagnosis. Baseline demographics and clinical characteristics are presented in Table 1.

\section{Univariate Analysis}

The median survival of our study cohort was 18.4 months (95\% CI, 16.3-20.5). With a median follow up time of 18.1 months (range, 2.0-112.1) the 1- and 2-year Kaplan-Meier estimates were as follows: loco-regional control 69.2\% (95\% CI, 58.0-80.4\%) and 28.0\% (95\% CI, 16.8-39.2\%), distant metastasis 52.9\% (95\% CI, 45.3-62.5\%) and 22.7\% (95\% CI, $15.4-30.0 \%$ ), overall survival $69.4 \%$ (95\% CI, 64.1-74.4\%) and 35.9\% (95\% CI, 30.4$41.4 \%$ ) [Table 2].

Increasing age was associated with increased all-cause mortality when assessed continuously (unadjusted HR 1.017, $p=0.003$ ). Treatment approach was significantly associated with survival on univariate analysis $(p<0.001)$ [Figure 1]. Improved survival rates were associated with resectable disease (2-year estimate $58.8 \%$ ) followed by borderline resectable disease (42.5\%), and finally unresectable disease $(16.2 \%, p<0.001)$ [Table 3]. Surgical resection was identified as a consistent significant parameter associated with survival (15.0 vs. $61.3 \%, p<0.001)$. Other oncologic treatment options, including immunotherapy ( 33.5 vs. $91.7 \%, p=0.012$ ) and chemotherapy ( 9.8 vs. $41.3 \%, p<0.001)$ were also associated with improved survival. Patient comorbidities were not associated with mortality.

Statin usage, both as prior ( 34.0 vs. $41.1 \%, p=0.021$ ) and active exposure (34.1 vs. $39.4 \%$, $p=0.030$ ) was associated with enhanced overall survival in our patient population (Figure 2). 
Furthermore, statin use correlated with increased 2-year overall survival in the subset of patients undergoing radiation therapy, surgery, and chemotherapy (55.0 vs. 84.1\%, $p<0.001$ ) [Table 4]. As expected, this finding was despite a greater proportion of patients with comorbidities, older mean age and lower use of chemotherapy among those receiving statins [Supplementary Table 1].

\section{Multivariable Analyses}

Variables that approached or achieved significance at the $p \unlhd) .10$ level were considered for multivariable Cox regression. Further analysis showed that both surgery and chemotherapy remained as significant independent prognostic factors [Table 5]. As statin use before or at diagnosis and active statin use were interacting variables, separate Cox regression models were calculated for each of these two variables. Additionally, to reduce the risk of immortal time bias associated with treatment arms, a conditional landmark analysis was conducted using a 6-month follow up cut off.

After applying a conditional landmark of 6-months, statin use before or at diagnosis was independently associated with all-cause mortality (adjusted HR 0.662, $p=0.027$ ), primarily by a reduction in the risk of distant metastasis (adjusted HR 0.577, $p=0.06$ ) [Figure 3]. Active statin use was similarly associated with a reduced risk of all-cause mortality following immortal time bias correction (adjusted HR 0.712, $p=0.049$ ), with a nonsignificant trend to reduction primarily in distant metastasis (adjusted HR 0.829, $p=0.444$ ).

\section{Discussion}

In the present analysis, we found that statin use before or at diagnosis was associated with a significantly reduced all-cause mortality (HR 0.662), primarily through a reduction in distant metastases (HR .577), in advanced-stage pancreatic cancer patients treated with stereotactic body radiotherapy. While active statin use had a similar correlation with overall survival, the strength of this association appeared less robust in comparison (HR 0.049). Based on these associations, statins may work to improve outcomes for advanced stage pancreatic cancer treatment.

These results are biologically plausible. By inhibiting the rate-limiting enzyme, statin usage results in an absolute reduction in downstream products of the mevalonate pathway. Namely, farynesyl diphosphate and geranylgeranyl diphosphate are downstream moieties required for prenylation and activation of Ras and Rho, respectively. ${ }^{4,18}$ A diminished flux through this biochemical pathway may result in reduced membrane association, activation, and proliferative effects of these GTP-binding proteins. Given that activating mutations in these proteins are known to be associated with $20-33 \%$ of all cancers, ${ }^{19,20}$ statins may be an effective complementary treatment option in malignancies with these phenotypes. Genomewide expression analysis has further revealed mutated tumor suppressor protein p53 to significantly upregulate the mevalonate pathway. ${ }^{21}$ While molecular subtyping is limited in directing pancreatic cancer treatment, expression of SMAD4, a signaling protein from transforming growth factor- $\beta$ (TGF- $\beta$ ) that enables cell cycle arrest and has tumor suppressor features, has been associated with significantly improvements in survival. ${ }^{22}$ As 
statins enhance the TGF- $\beta /$ SMAD pathway, thereby promoting apoptosis, statins may be most efficacious in pancreatic cancers that express SMAD.

Interestingly, statin use in these malignancies has been shown to mimic the phenotype of mutant p53 depletion, and as discussed above, statins seem to obstruct cell cycle progression, arresting cells in G1, suggesting they may exert potent radiosensitizing effects. 23-25 All patients in this cohort received radiation therapy, thus statin-induced radiosensitization of the pancreatic cancer cells was possible. Finally, we found comparable evidence for statin usage improving survival both prior to and after diagnosis. These findings potentially support both a systemic effect and synergy with radiotherapy and chemotherapy as potential mechanisms by which statins may enhance survival in this cohort.

HMG-CoA reductase inhibitors selectively act in the liver by reducing hepatic cholesterol biosynthesis, thus resulting in enhanced LDL receptor turnover and ultimately decreased plasma LDL concentration. As statins primarily localize in the liver, it seems possible that the primary anti-tumor effects may be via lowering serum cholesterol and depriving rapidly dividing malignant cells of necessary constituents for proliferation. Low serum cholesterol, not entirely explained by poor nutrition, has been observed in cancer patients. ${ }^{26}$

Accordingly, the systemic effects associated with statin use remains a viable hypothesis for improving cancer-specific outcomes at extra-hepatic sites. As a result, there are currently 36 trials testing the efficacy of statins to improve outcomes in several cancer subsites (clinicaltrials.gov).

Improvement in outcomes in the treatment of other cancer subsites are associated with statin use, including breast ${ }^{27}$ and prostate cancer. ${ }^{28}$ However, a recent literature review yielded only two previous studies evaluating the impact of statins on survival in pancreatic cancer patients. In a 226-patient cohort, Wu et al. reported both prior and active simvastatin usage to be associated with improved survival in early-stage pancreatic cancer patients undergoing resection. ${ }^{13}$ Though they reported a stronger association between survival and statin usage, our data is consistent with these results. Given the cohort of the preceding study was entirely comprised of early-stage disease in comparison to our advanced-stage population, it seems feasible that less advanced cancers with longer overall survival times may generate a more noticeable effect.

Furthermore, research failing to describe a relationship between statin treatment and survival in other cancer sites has speculated that flawed study design may yield erroneous results in previous statin-survival analyses. ${ }^{29}$ Emilsson et al. cite confounding, selection, and immortal-time bias as possible threats to the validity of previous data. The risk of confounding bias is inherent to observational studies, however, we accounted for this possibility by considering any variable with $p<0.10$ for inclusion in our multivariable model and providing a conditional landmark to avoid immortal time bias associated with treatment arms. Additionally, we desired to assess time dependence of statin use. While this could not be evaluated on a continuous time scale due to concerns about medical record accuracy/ availability of the exact date of initiating statins, we were able to identify differences in those exposed to statins within a 6-month window of treatment and those only using statins during treatment. As discussed previously, those exposed prior to and at diagnosis appeared 
to have a more robust association with survival benefit, indicating separate potential systemic and synergistic effects with radiotherapy. This benefit was upheld even when accounting for immortal time bias that could skew the influences of other treatment arms in multivariable analysis.

This retrospective work has several limitations including heterogeneity of the sample group. However, this did allow for a larger cohort that enabled accounting for the varying treatment modalities and their impact on outcomes. Statin dosages were rarely available for collection, and we were therefore unable to establish a dose-response relationship which may have reinforced our findings. However, large analyses have failed to describe a dose-response relationship, indicating that the impact of statins on survival may be a binary relationship. ${ }^{11}$ We were unable to investigate the relationship between individual statin agents and outcomes since prescribed statin medications varied and the heterogeneous nature of the prescribed statins left small numbers for individual analysis. It is possible that characteristics of individual statin medications such as lipophilicity may influence outcomes in cancer patients and represents an area of future research. Due to limitations with access to records among all patients indicating the exact start of statin use (i.e. records from the prescribing physician), we could not assess exact time points and their influence on outcome gains seen. Finally, patient data was acquired retrospectively, and unavoidable confounding is possible.

\section{Conclusions}

To our knowledge this is the first study to analyze how statin therapy may affect survival, loco-regional failure, and distant metastasis in advanced-stage pancreatic adenocarcinoma. In the present study, we report an association between statin use and cancer-specific outcomes, revealing the efficacy of statin use as an ancillary cancer treatment option. Future prospective studies are needed before statins can be justified for off-label usage against pancreatic cancer.

\section{Supplementary Material}

Refer to Web version on PubMed Central for supplementary material.

\section{Acknowledgments}

Funding: Research reported in this publication was supported by the National Institute of Health grant award number T35DK065521.

\section{References}

1. Miller KD, et al. Cancer treatment and survivorship statistics, 2016. CA Cancer J Clin. 66:271289.2016; [PubMed: 27253694]

2. Katz MHG, et al. Borderline Resectable Pancreatic Cancer: The Importance of This Emerging Stage of Disease. J Am Coll Surg. 206:833-846.2008; [PubMed: 18471707]

3. Mishra MV, et al. Conditional Survival Probabilities for Patients With Resected Pancreatic Adenocarcinoma. Am J Clin Oncol. 37:107-111.2012;

4. Chan KKW, Oza AM, Siu LL. The statins as anticancer agents. Clin Cancer Res. 9:10-9.2003; [PubMed: 12538446] 
5. Warita K, et al. Statin-induced mevalonate pathway inhibition attenuates the growth of mesenchymal-like cancer cells that lack functional E-cadherin mediated cell cohesion. Sci Rep. 4:7593.2015;

6. Pelaia G, et al. Effects of statins and farnesyl transferase inhibitors on ERK phosphorylation, apoptosis and cell viability in non-small lung cancer cells. Cell Prolif. 45:557-565.2012; [PubMed: 23045963]

7. Qi XF, et al. HMG-CoA reductase inhibitors induce apoptosis of lymphoma cells by promoting ROS generation and regulating Akt, Erk and p38 signals via suppression of mevalonate pathway. Cell Death Dis. 4:e518.2013; [PubMed: 23449454]

8. Dale KM, Coleman CI, Henyan NN, Kluger J, White CM. Statins and Cancer Risk. JAMA. 295:74.2006; [PubMed: 16391219]

9. Bonovas S, Filioussi K, Tsavaris N, Sitaras NM. Statins and cancer risk: a literature-based metaanalysis and meta-regression analysis of 35 randomized controlled trials. J Clin Oncol. 24:480817.2006; [PubMed: 17001070]

10. Murtola TJ, Visvanathan K, Artama M, Vainio H, Pukkala E. Statin Use and Breast Cancer Survival: A Nationwide Cohort Study from Finland. PLoS One. 9:e110231.2014; [PubMed: 25329299]

11. Nielsen SF, Nordestgaard BG, Bojesen SE. Statin Use and Reduced Cancer-Related Mortality. N Engl J Med. 367:1792-1802.2012; [PubMed: 23134381]

12. Gutt R, et al. Statin use and risk of prostate cancer recurrence in men treated with radiation therapy. J Clin Oncol. 28:2653-9.2010; [PubMed: 20421534]

13. Wu BU, et al. Impact of statin use on survival in patients undergoing resection for early-stage pancreatic cancer. Am J Gastroenterol. 110:1233-9.2015; [PubMed: 26195180]

14. Jeon CY, et al. The Association of Statin Use after Cancer Diagnosis with Survival in Pancreatic Cancer Patients: A SEER-Medicare Analysis. PLoS One. 10:e121783.2015; [PubMed: 25830309]

15. Chubak J, Boudreau DM, Wirtz HS, McKnight B, Weiss NS. Threats to Validity of Nonrandomized Studies of Postdiagnosis Exposures on Cancer Recurrence and Survival. JNCI J Natl Cancer Inst. 105:1456-1462.2013; [PubMed: 23940288]

16. Cardwell CR, Hicks BM, Hughes C, Murray LJ. Statin Use After Colorectal Cancer Diagnosis and Survival: A Population-Based Cohort Study. J Clin Oncol. 32:3177-3183.2014; [PubMed: 25092779]

17. Eisenhauer EA, et al. New response evaluation criteria in solid tumours: Revised RECIST guideline (version 1.1). Eur J Cancer. 45:228-247.2009; [PubMed: 19097774]

18. Jackson SM, Ericsson J, Edwards PA. Signaling molecules derived from the cholesterol biosynthetic pathway. Subcell Biochem. 28:1-21.1997; [PubMed: 9090289]

19. Downward J. Targeting RAS signalling pathways in cancer therapy. Nat Rev Cancer. 3:1122.2003; [PubMed: 12509763]

20. Vigil D, Cherfils J, Rossman KL, Der CJ. Ras superfamily GEFs and GAPs: validated and tractable targets for cancer therapy? Nat Rev Cancer. 10:842-57.2010; [PubMed: 21102635]

21. Freed-Pastor WA, et al. Mutant p53 Disrupts Mammary Tissue Architecture via the Mevalonate Pathway. Cell. 148:244-258.2012; [PubMed: 22265415]

22. Xia X, et al. SMAD4 and its role in pancreatic cancer. Tumor Biol. 36:111-119.2015;

23. Lacerda L, et al. Simvastatin radiosensitizes differentiated and stem-like breast cancer cell lines and is associated with improved local control in inflammatory breast cancer patients treated with postmastectomy radiation. Stem Cells Transl Med. 3:849-56.2014; [PubMed: 24833589]

24. He Z, et al. Cell killing and radiosensitizing effects of atorvastatin in PC3 prostate cancer cells. J Radiat Res. 53:225-33.2012; [PubMed: 22510595]

25. Rao S, Lowe M, Herliczek TW, Keyomarsi K. Lovastatin mediated G1 arrest in normal and tumor breast cells is through inhibition of CDK2 activity and redistribution of p21 and p27, independent of p53. Oncogene. 17:2393-402.1998; [PubMed: 9811471]

26. Kritchevsky SB, Kritchevsky D. Serum cholesterol and cancer risk: an epidemiologic perspective. Annu Rev Nutr. 12:391-416.1992; [PubMed: 1503812] 
27. Ahern TP, et al. Statin Prescriptions and Breast Cancer Recurrence Risk: A Danish Nationwide Prospective Cohort Study. JNCI J Natl Cancer Inst. 103:1461-1468.2011; [PubMed: 21813413]

28. Zaorsky NG, Buyyounouski MK, Li T, Horwitz EM. Aspirin and Statin Nonuse Associated With Early Biochemical Failure After Prostate Radiation Therapy. Int J Radiat Oncol. 84:e13-e17.2012;

29. Emilsson L, et al. Examining Bias in Studies of Statin Treatment and Survival in Patients With Cancer. JAMA Oncol. 7:e29849.2017; 


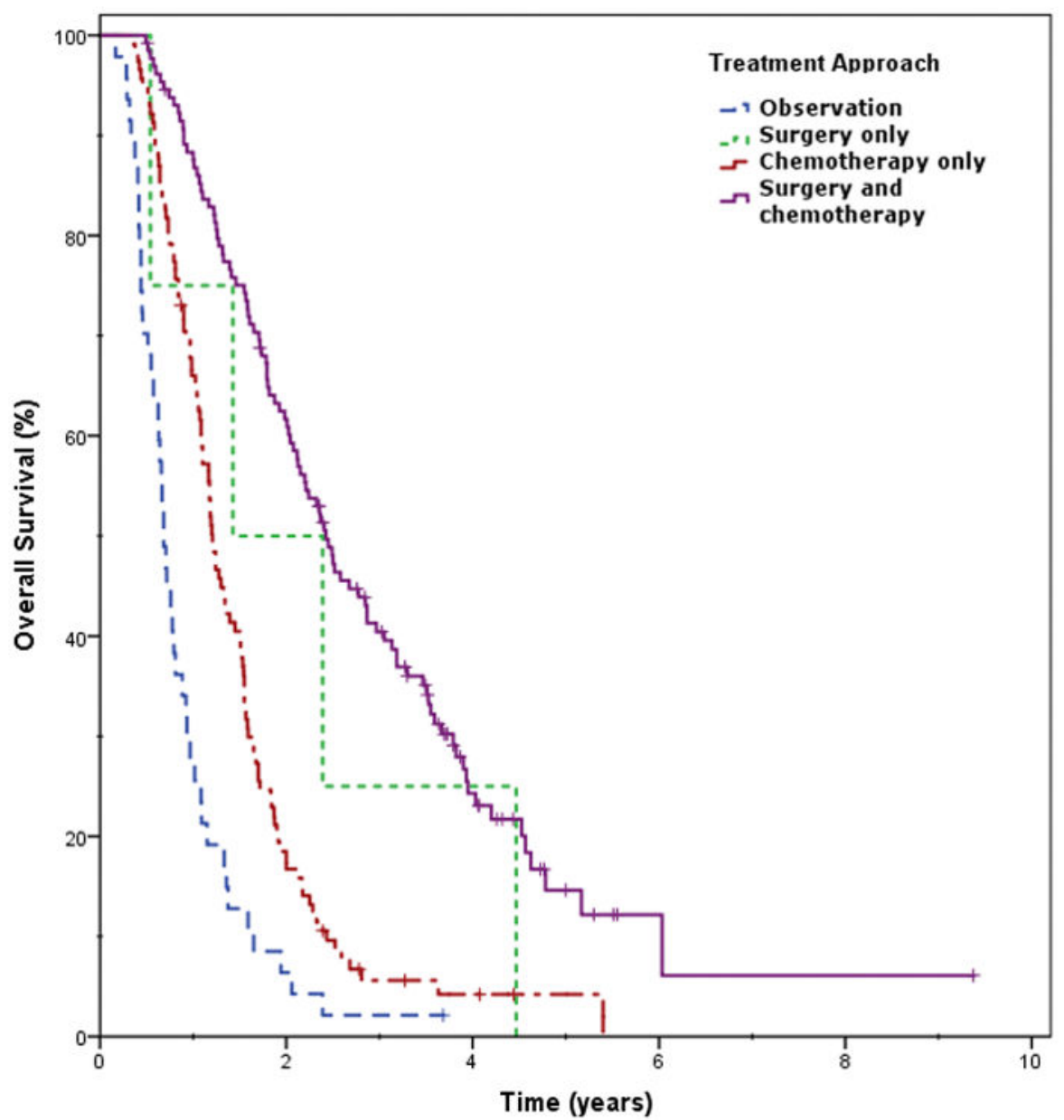

Figure 1.

Overall survival based on treatment approach (univariate analysis, $\mathrm{p}<0.001$ ) with surgery and chemotherapy independently improving outcomes. 

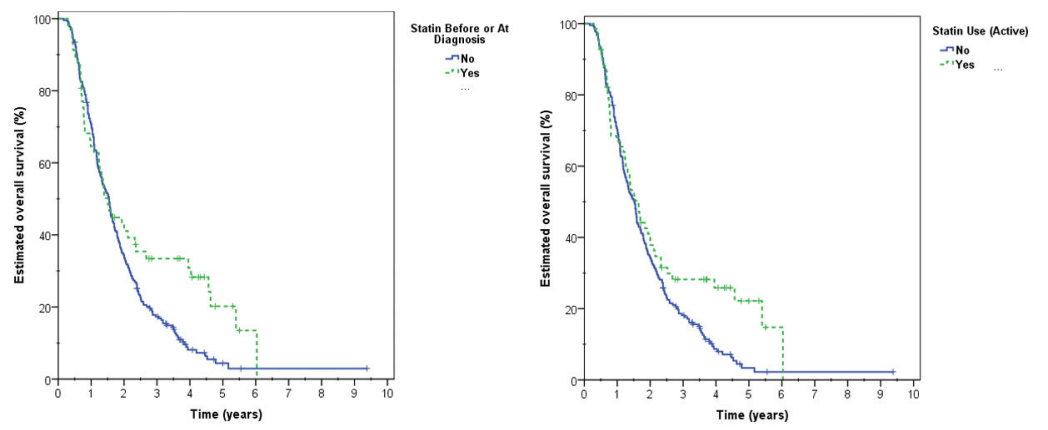

Figure 2.

Unadjusted overall survival estimates based on statin use before or at diagnosis $(\mathrm{p}=0.021)$ and active statin use during treatment $(\mathrm{p}=0.030)$. 

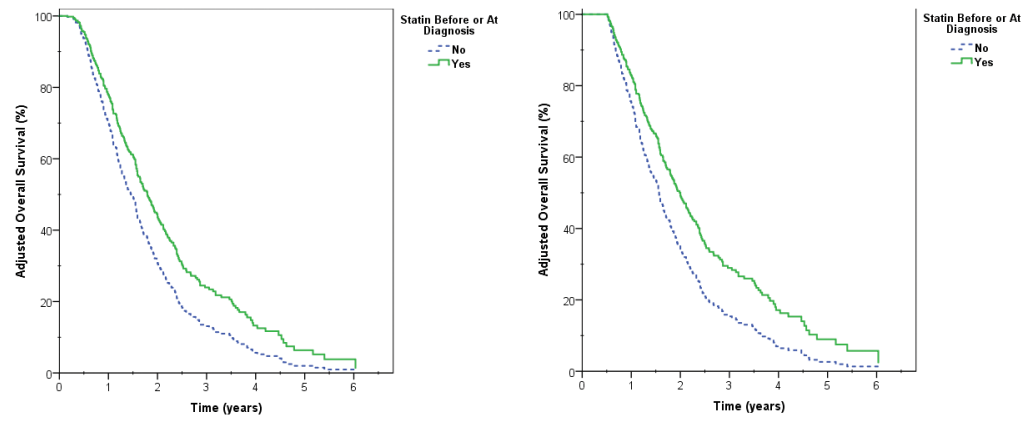

Figure 3.

Adjusted overall survival estimate based on statin use before or at diagnosis without (left) and with (right) immortal time bias adjustment, defined as exclusion of patients with $<6$ months follow up. 
Table 1

Patient, disease and treatment characteristics $(n=303)$ - excluded non-adenocarcinoma $(n=5)$

\begin{tabular}{|c|c|}
\hline \multicolumn{2}{|l|}{ Patient characteristics } \\
\hline Age, years & \\
\hline Median (range) & $70(33-90)$ \\
\hline \multicolumn{2}{|l|}{ Gender } \\
\hline Male & $152(50.2 \%)$ \\
\hline Female & $151(49.8 \%)$ \\
\hline \multicolumn{2}{|l|}{ Race } \\
\hline Caucasian & $277(91.4 \%)$ \\
\hline Other & $12(4.0 \%)$ \\
\hline Unknown & $14(4.6 \%)$ \\
\hline \multicolumn{2}{|l|}{ Body mass index } \\
\hline Median (range) & $24.8(14.5-45.8)$ \\
\hline \multicolumn{2}{|l|}{ Comorbidities } \\
\hline Diabetes & $176(58.1 \%)$ \\
\hline Coronary artery disease & $37(12.2 \%)$ \\
\hline Hyperlipidemia & $73(24.1 \%)$ \\
\hline Hypertension & $207(68.3 \%)$ \\
\hline \multicolumn{2}{|l|}{ Medications } \\
\hline Metformin & $41(13.5 \%)$ \\
\hline Pancreatic enzymes & $130(42.9 \%)$ \\
\hline Sulfonylurea & $23(7.6 \%)$ \\
\hline Insulin & $158(52.1 \%)$ \\
\hline Statin (active use during treatment) & $71(23.4 \%)$ \\
\hline Statin (before or at diagnosis) & $59(19.5 \%)$ \\
\hline Glucocorticoid & $91(30.0 \%)$ \\
\hline Beta-blocker (before or at diagnosis) & $53(17.5 \%)$ \\
\hline Beta-blocker (active use) & $107(35.3 \%)$ \\
\hline \multicolumn{2}{|l|}{ Disease characteristics } \\
\hline \multicolumn{2}{|l|}{ Histology } \\
\hline Adenocarcinoma & $303(100 \%)$ \\
\hline \multicolumn{2}{|l|}{ Lymph node status } \\
\hline cNO & $127(41.9 \%)$ \\
\hline $\mathrm{cN}+$ & $164(54.1 \%)$ \\
\hline Not reported & $12(4.0 \%)$ \\
\hline
\end{tabular}

Am J Clin Oncol. Author manuscript; available in PMC 2019 September 05. 


\begin{tabular}{|c|c|c|}
\hline \multirow{8}{*}{ 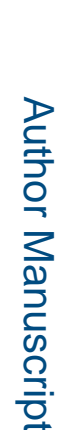 } & Unresectable & $149(49.2 \%)$ \\
\hline & Borderline resectable & $40(13.2 \%)$ \\
\hline & Resectable & $111(36.6 \%)$ \\
\hline & Unknown & $3(1.0 \%)$ \\
\hline & \multicolumn{2}{|c|}{ Treatment characteristics } \\
\hline & Surgery & \\
\hline & Yes & $136(44.9 \%)$ \\
\hline & No & $167(55.1 \%)$ \\
\hline & \multicolumn{2}{|l|}{ Immunotherapy } \\
\hline & Yes & $12(4.0 \%)$ \\
\hline & No & $291(96.0 \%)$ \\
\hline & Chemotherapy & \\
\hline$\underline{D}$ & Yes & $248(81.8 \%)$ \\
\hline วิ & No & $55(18.2 \%)$ \\
\hline
\end{tabular}

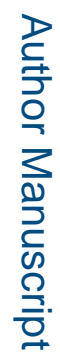

로을

Am J Clin Oncol. Author manuscript; available in PMC 2019 September 05. 
Table 2

Kaplan-Meier estimated outcomes with a median follow-up of 18.1 months (range 2.0-112.1)

\begin{tabular}{|l|c|c|}
\hline & 1-year estimate (95\% CI) & 2-year estimate (95\% CI) \\
\hline Locoregional control & $69.2 \%(58.0-80.4 \%)$ & $28.0 \%(16.8-39.2 \%)$ \\
\hline Distant metastasis rate & $52.9 \%(45.3-62.5 \%)$ & $22.7 \%(15.4-30.0 \%)$ \\
\hline Overall survival & $69.4 \%(64.1-74.7 \%)$ & $35.9 \%(30.4-41.4 \%)$ \\
\hline
\end{tabular}




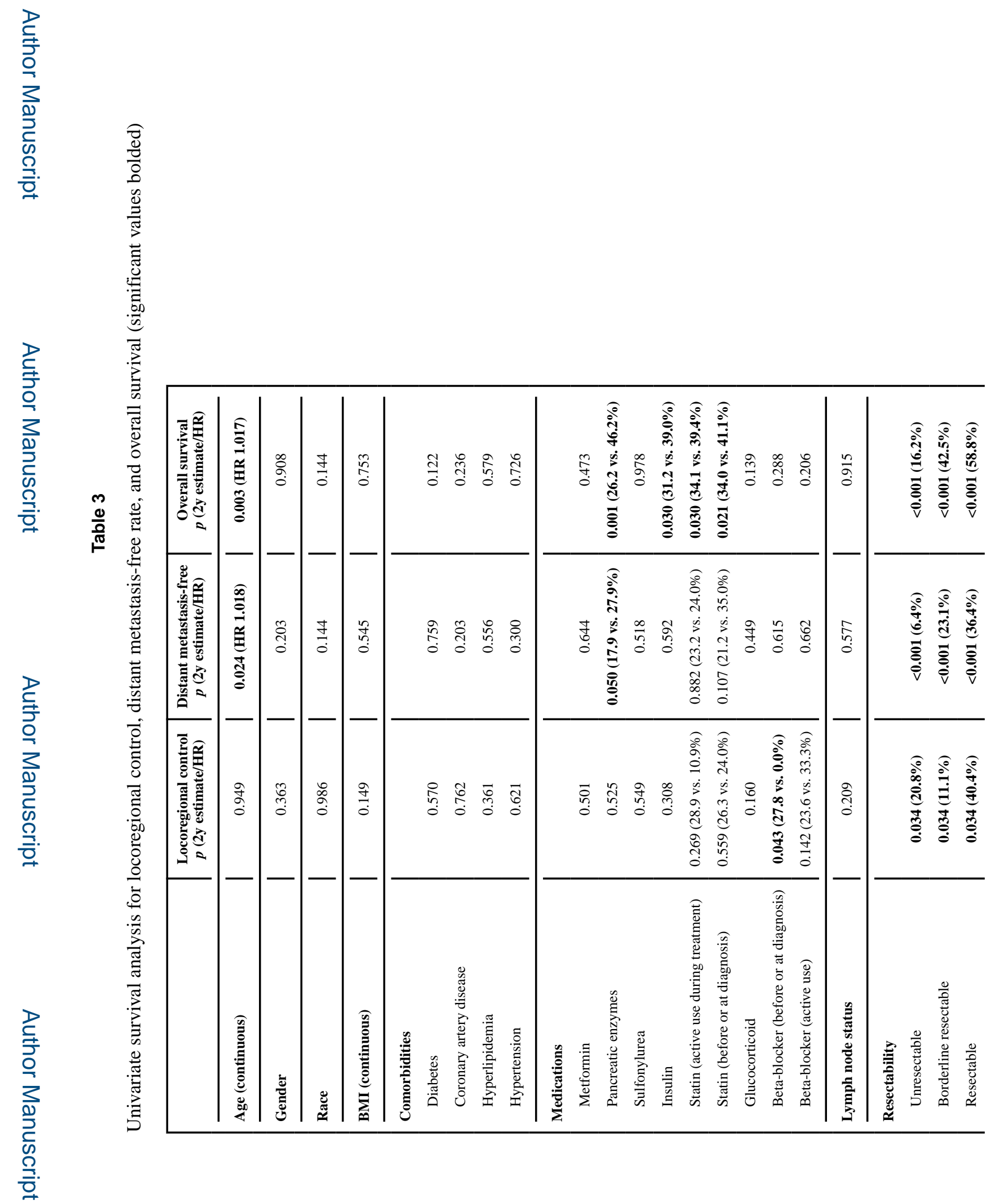

Am J Clin Oncol. Author manuscript; available in PMC 2019 September 05. 


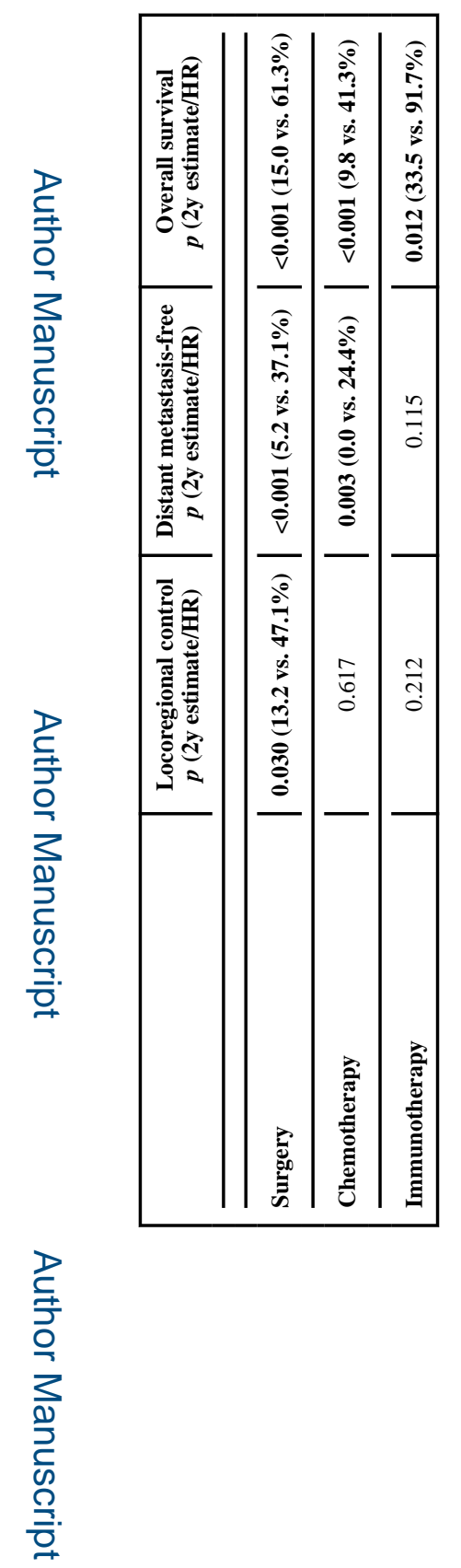

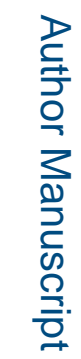




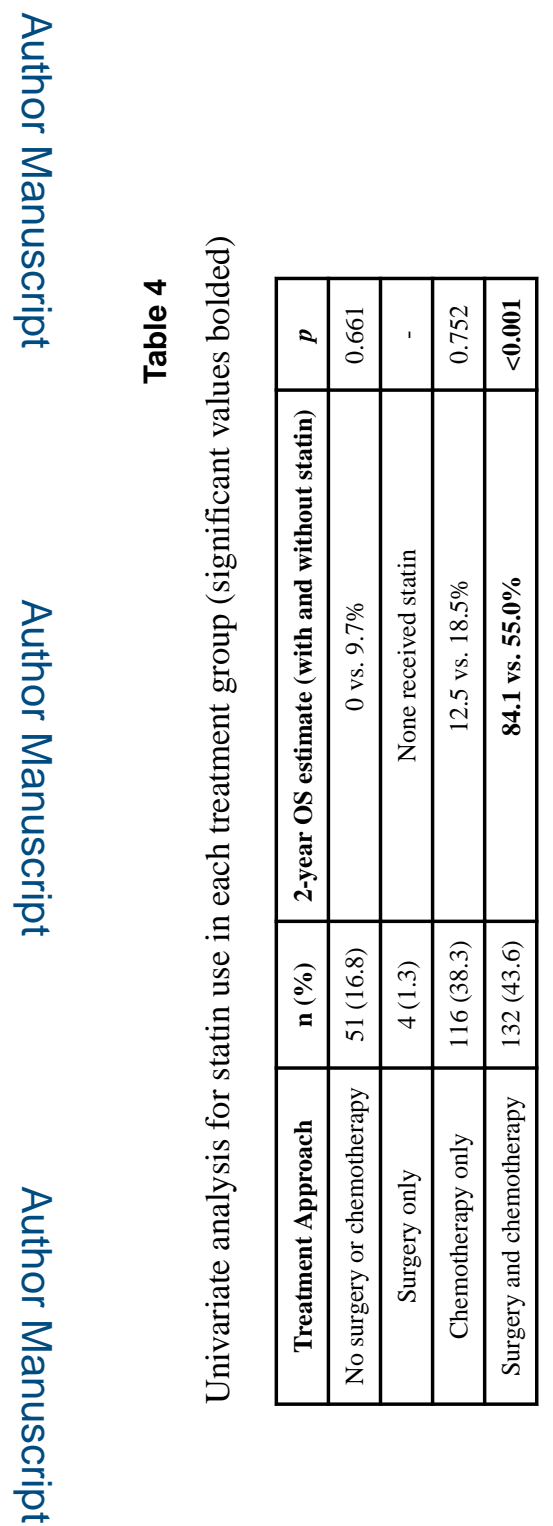

Am J Clin Oncol. Author manuscript; available in PMC 2019 September 05. 


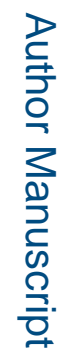

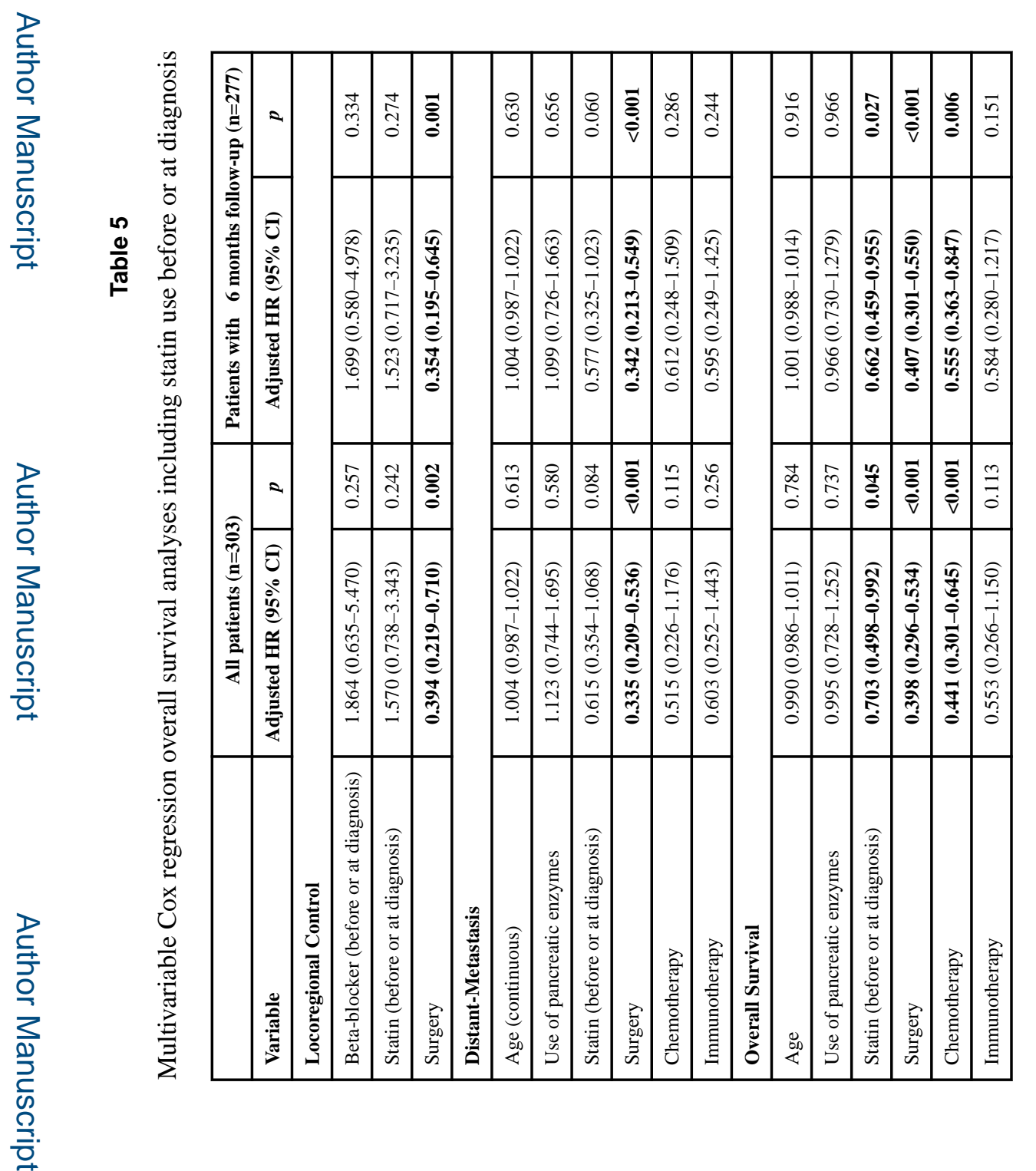

Am J Clin Oncol. Author manuscript; available in PMC 2019 September 05. 